\title{
Economía y trabajo en equipo como fuente de ventaja competitiva en las aulas de Secundaria
}

* Paula Díaz de la Buelga

\author{
PALABRAS CLAVE \\ Economía \\ Motivación \\ Trabajo en equipo \\ Grupo de trabajo \\ Educación
}

\begin{abstract}
RESUMEN
El trabajo en equipo es un término muy utilizado hoy en día en las organizaciones y deriva de múltiples teorías económicas que han sido objeto de estudio durante años por diversas personas economistas, sociólogas y filósofas. Sus beneficios están respaldados por numerosas investigaciones de campo que prueban la efectividad de estas técnicas en el ámbito laboral y los beneficios que reporta en términos de productividad. Pese a que la mayor parte de los estudios están realizados en el ámbito de la empresa y las organizaciones, en los últimos años, estas teorías han sido objeto de aplicación en otros ámbitos, entre ellos, el educativo. El trabajo en equipo es una competencia que cada vez está más presente en nuestras vidas, por ello, los alumnos y alumnas deben de comenzar a potenciar sus habilidades para el trabajo en equipo desde edades tempranas y en todas las fases del sistema educativo. Fomentar el trabajo autónomo y colaborativo, así como crear actividades susceptibles de fortalecer la competencia del trabajo en equipo es una labor que como docentes tenemos la obligación de perseguir. Por ello, en el presente trabajo se realiza una propuesta educativa para dinamizar las sesiones de clase, con la intención de enseñar a los alumnos y alumnas a aprender a aprender a través del trabajo en equipo.
\end{abstract}

\section{Economy and teamwork as a source of competitive advantage in Secondary classrooms}

\begin{abstract}
Nowadays, teamwork is a widely used term in organizations, and it derives from multiple economic theories that have been studied for years by various economists, sociologists and philosophers. Its benefits are supported by numerous field investigations that prove the effectiveness of these techniques in the workplace and the benefits it brings in terms of productivity. Although most of the studies are carried out in the field of business and organizations, in recent years, these theories have been applied in other areas, including education. Teamwork is a competition that is increasingly present in our lives and in the workplace around us. Therefore, students must begin to enhance their teamwork skills from an early age and at all stages of the education system. Fostering autonomous and collaborative work as well as creating activities that can strengthen the competence of teamwork, is a task that as teachers we have an obligation to pursue. For this reason, in this work an educational proposal is made to stimulate class sessions, with the intention of teaching students to learn to learn through teamwork.
\end{abstract}

Universidad de Oviedo

Autora de correspondencia: * Paula Díaz de la Buelga; paulabuelga@hotmail.com

Recibido: 30/07/2020 - Aceptado: 30/03/2021

Revista de Formación del Profesorado e Investigación Educativa

Facultad de Formación del Profesorado y Educación

Universidad de Oviedo

Julio - Diciembre 2020

ISSN: 2340-4728 


\section{Introducción}

En nuestra vida diaria, las rutinas se olvidan rápidamente y pasan inadvertidas. Sin embargo, cuando pasa algo distinto y que nos coge por sorpresa, suele recordarse con más facilidad. Esto mismo sucede en el ámbito académico. Los alumnos y alumnas recuerdan con más intensidad las experiencias que perciben como diferentes, por tanto, podemos decir que, muchas veces, la sorpresa nos ayuda a aprender.

Las técnicas experimentales y los juegos en el aula, además de captar la atención del alumnado y despertar su curiosidad, pueden potenciar su interés y permitir que afiancen conceptos y que los entiendan mejor. Aun así, es fundamental combinar varias técnicas de enseñanza-aprendizaje, incluidas las tradicionales.

Numerosos estudios avalan la importancia del trabajo en equipo, no sólo a nivel de mejora del rendimiento del grupo y la productividad, sino también a nivel personal, anímico y emocional de las personas.

El mismo Howard Gardner en su Teoría de las Inteligencias Múltiples manifiesta cómo trabajar en grupo o mediante equipo de trabajo genera sinergias positivas que hacen que "el todo sea mayor que la suma de las partes" (Suárez et al., 2010).

Lograr que un estudiante mejore su autoestima se reflejará en su aprovechamiento escolar y desarrollo personal, así como una vez titulado será capaz de afrontar sus tareas profesionales con ética y responsabilidad (Mejía et al., 2011).

En este sentido cabe citar a Ferrán (2015, p. 231), cuando manifiesta:

La obligatoriedad de la enseñanza secundaria ofrece la oportunidad de incluir dentro de las actividades escolares programas optativos orientados a mejorar los estilos y estrategias de afrontamiento, a unas edades en las que el bienestar personal disminuye de manera significativa. Con ello se puede fomentar no sólo el bienestar sino también prevenir alteraciones de tipo emocional ligadas a los cambios sociales, físicos y psicológicos que caracterizan a este grupo de edad. Cabe recordar, como señalan Bermúdez, Teva y Buela- Casal (2009), que fomentar un afrontamiento productivo de las situaciones estresantes constituye un componente clave de los programas de prevención primaria y de promoción de la salud.

Por otro lado, desde el punto de vista de la empresa y las organizaciones y citando a Pérez, Pineda y Arango (2011, p 10):

El diseño de las nuevas organizaciones invita a crear nuevas formas de organización más sistémicas (Senge, 1993, p. 164175) y flexibles, concepto que ya vislumbraba Drucker a finales del siglo XX para las compañías manufactureras (1995, p. 295-312). Este tipo de organizaciones posibilita aprovechar las destrezas de cada trabajador, según Goshall \& Barlett (1999) y genera organizaciones paralelas fundamentadas en el trabajo en equipo (Gryna, Chua y Defeo, 2007, p. 214-216) o por proyectos (Gilli, 2007, p. 168-169).

A pesar de que en las empresas coexisten los grupos de trabajo, en diferentes niveles jerárquicos y con dinámicas diversas, el trabajo en equipo es diferente: "En el trabajo en equipo se fijan responsabilidades (en particular, se asigna carga de trabajo) a los equipos como tales. Se calculan los resultados del individuo y del equipo, y a veces solo los del equipo. El hecho de trabajar en equipo implica un cierto grado de control y apoyo mutuo, tanto mayor como más importantes sean los resultados globales para sus componentes" (Cuatrecasas, 2005$, p. 7$)$.

Para poder hablar de trabajo en equipo, debemos desgranar este concepto y determinar qué es trabajo en equipo y qué no lo es. Pues, en ocasiones, se confunden los términos y se asume por defecto que trabajo en grupo y trabajo en equipo son sinónimos, cuando no es así. De hecho, nos encontramos con que los estudiantes y las estudiantes creen que trabajan muy bien en equipo y tienen claro que saben trabajar en equipo. Sin embargo, creen que trabajar en equipo implica que un grupo de personas hagan un trabajo, repartiéndose los puntos de elaboración del mismo y, luego "cortando y pegando" las partes en un solo documento. El trabajo en equipo constituye una herramienta esencial para abordar tareas cada vez más complejas en un entorno siempre cambiante. El problema, a nivel educativo, se centra en cómo conseguir que el trabajo en equipo sea eficaz, es decir, en cómo conseguir un trabajo que sea verdaderamente de equipo.

Por ello, "es imprescindible llegar a un consenso que favorezca una consonancia de las sinergias educativas para facilitar la incorporación al mundo laboral" (Barraycoa y Lasaga, 2010). La labor pedagógica debe encaminarse a que el alumnado entienda que trabajar en equipo no es una mera adición de esfuerzos, a que entienda que el trabajo en equipo requiere de poner en marcha habilidades conjuntas como la comunicación interpersonal, la responsabilidad y compromiso con los miembros del equipo, la gestión del tiempo, la confianza y el respeto.

Trabajar en equipo consiste en colaborar organizadamente para obtener un objetivo común. Cada miembro del equipo tiene una aptitud y posición determinadas. Todas las personas han de manifestarse y relacionarse entre sí coordinadamente en pos del objetivo. Todas son importantes para la eficacia del trabajo en equipo. Trabajar en equipo implica aportar valor añadido específico al equipo, ser adaptable y gestionar bien el tiempo (Cardona y Wilkinson, 2006). Además, implica conocimiento.

Citando a Marín-García y Zarate-Martínez (2008, p. 257),

El conocimiento ha sido calificado como uno de los recursos más valiosos en la sociedad actual (Davenport y Prusak, 1998; Holsapple y Yoshi, 2002; Martínez, 2006; Nonaka y Takeuchi, 1995; OECD, 1996; Reyes, 2005) y constituye un aliado en la eficiencia de los métodos de producción y organización (perfectamente trasladable al ámbito de la educación), así como en la mejora de productos y servicios (Wiig, 1997), por lo que se hace necesaria su gestión

Es necesario que todos los componentes y las componentes del equipo contribuyan de manera proporcional a sus capacidades. De lo contrario, aparece el fenómeno de los free riders o polizones, esto es, personas que se benefician a costa del esfuerzo de sus compañeros y compañeras. (Marín-García y Zarate, 2008).

Así mismo, el trabajo en equipo, en ocasiones, implica división de las tareas a realizar y especialización del trabajo. Adam Smith fue el primer economista en hablar de la división del trabajo en su teoría del Crecimiento Económico, que constituye el interés central en su análisis de la Riqueza de las Naciones. Ricoy (2005, p.12) expone que el núcleo del análisis de Smith del progreso o crecimiento económico está compuesto por:

a) Su concepción de la acumulación de capital y de la división del trabajo.

b) La proposición de que la "división del trabajo está limitada por el alcance o la extensión del mercado" y esta, a su vez, está basada en la relación de dependencia recíproca existente entre la extensión y generalización de la división social del trabajo (del proceso de subdivisión y especialización de industrias) y la intensidad y el desarrollo del proceso de intercambio.

c) El argumento de que todo acto de ahorro supone siempre (aunque no necesariamente de forma simultánea), una decisión o un acto correlativo de inversión, es decir, de acumulación de capital. Este último argumento es equivalente, y antecedente, a la ley de Say.

El avance de la tecnología, la globalización, una mano de obra más especializada, más crítica respecto al valor de su tiempo y la dependencia del mercado fuerzan a las organizaciones a hacer 
más con menos. El objetivo es aprovechar al máximo su capital humano y adaptarse a las exigencias del entorno mediante el uso de grupos de personas que realicen actividades que resultarían muy complejas para llevarlas a cabo con éxito de forma individual (Marín-García y Zarate, 2008). En este sentido, se justifica la importancia de enseñar desde edades tempranas y en todos los niveles educativos qué es trabajar en equipo y cómo se trabaja en equipo.

Sin embargo, la utilización del trabajo en equipo puede enfocarse, además de desde esta perspectiva instrumental, concibiendo el trabajo en equipo como una herramienta al servicio de la producción, desde una perspectiva sociológica, centrada en la persona y en su bienestar en el trabajo (Marín-García y Zarate Martínez, 2008). En relación con esta última perspectiva, tendría sentido atender a la conexión existente entre motivación y trabajo en equipo.

Un estudio realizado en 2015 por la Escuela Nacional de Salud Pública de Cuba pone de manifiesto este estrecho vínculo y cómo la interacción permanente del hombre y su medio de trabajo físico y psicológico influye sobre su salud positiva o negativamente y sobre su rendimiento y resultados de trabajo (Hernández y Hernández, 2015). Según esta investigación, el participar en un equipo da a los trabajadores y trabajadoras mayor control en la tarea de mejorar la calidad, lo que lleva a una mayor satisfacción, motivación y eficiencia. Por lo tanto, se observó la relación que guardan la motivación y el trabajo en equipo. Los trabajadores que participan en equipos se motivan por la experiencia de trabajar con sus compañeros y compañeras en la búsqueda de maneras de mejorar sistemas y procesos de la empresa y resolver problemas. Si el equipo logra alcanzar sus metas, la experiencia suele ser muy satisfactoria y genera una fuerte identificación con el equipo y orgullo por sus logros (Hernández y Hernández, 2015).

La motivación es un elemento que afecta directamente sobre la eficacia de las organizaciones, y que en muchas ocasiones se ve afectado por la existencia del estrés. Es en los años setenta cuando aparece por primera vez el concepto de Burnout, pero no será hasta los años ochenta cuando se produzca un aumento considerable de las investigaciones sobre este síndrome (Herrazo y Moriana, 2004).

Hoy en día, los altos niveles de estrés a los que estamos expuestos y las nuevas exigencias de la sociedad actual en la que vivimos, tanto a nivel laboral como académico y especialmente en los países industrializados, han originado la aparición de nuevos riesgos denominados psicosociales. Entre ellos, destaca el conocido síndrome de Burnout, o síndrome del trabajador quemado, que tiene por características principales el agotamiento emocional, la despersonalización o deshumanización y la falta de realización personal en el trabajo, y cuya prevalencia se ha ido incrementando en los últimos años.

El síndrome de Burnout ha llegado a convertirse, en ocasiones, en un problema social y de salud pública que conlleva, por tanto, un gran coste económico y social, y por ello, son numerosos los estudios que se han realizado en los últimos años al respecto (Gil, 2005).

El síndrome de Burnout ha sido desarrollado mayormente en el ámbito laboral, pero el campo de estudio es muy amplio y existen estudios aplicados a entornos educativos. En este sentido, cabe mencionar un estudio efectuado en 2009 en la ciudad de Durango (México) que revela unos niveles de burnout leves y moderados en el estudiantado de secundaria. Estos resultados resultan preocupantes por la edad y etapa en la que viven los sujetos encuestados y que son objeto del análisis (Gutiérrez, 2009). Desde la perspectiva docente, un estudio realizado en ese mismo año por la Universidad Internacional de Cataluña revela en nuestro cuerpo docente un perfil de Burnout con niveles moderados o medios en la muestra de docentes analizados. Además, se detectó la existencia de un porcentaje elevado de docentes a los que podríamos considerar susceptibles de padecer Burnout (Arís, 2009).
En resumen, numerosos autores y autoras defienden el potencial y las ventajas del trabajo en equipo, siendo este considerado "un punto clave y una ventaja competitiva (Badger, Sadler-Smith et Michie, 1997; Rousseau, Aubé, et Savole, 2006; Tjosvold, 1991), además de ser una competencia participativa que permite aumentar la productividad, la innovación y la satisfacción en el trabajo" (Torrelles et al., 2011, p.).

\section{Objetivos}

El objetivo del presente trabajo es presentar una propuesta docente que, utilizando el trabajo en equipo como metodología docente, implique la dinamización de las clases a través de la inclusión de experiencias innovadoras y juegos en las sesiones de aula.

\section{Método}

\section{Participantes}

Como el objetivo del trabajo es presentar una propuesta docente, no se cuenta con información objetiva sobre la muestra o participantes con los que trabajar. Aun así, la propuesta se ha diseñado teniendo en mente un aula tipo, de unos 24 alumnos y alumnas, divididos en equipos de trabajo de, como máximo, 4 personas, de Educación Secundaria Obligatoria.

\section{Temporización}

El diseño de la propuesta se ha realizado teniendo en mente un curso académico, aunque sería posible ajustarlo por trimestres.

\section{Metodología}

El trabajo en equipo será la base de trabajo sobre la que se diseña la propuesta. Esta metodología va dirigida a fomentar el trabajo autónomo del alumnado y, sobre todo, a potenciar las habilidades personales, que son las que contribuyen a enriquecer el trabajo con otros compañeros/as para, finalmente, lograr un auténtico trabajo en equipo, dando visibilidad a la fuerza colectiva de la unidad.

\section{Procedimiento}

Esta propuesta docente está diseñada para ser implantada en la asignatura de Economía de $1^{0}$ de Bachillerato, por su relación directa con los contenidos que en ella se imparten. Esta asignatura, que cuenta con cuatro horas lectivas a la semana, es una de las materias troncales incluida en el Anexo I del Decreto 42/2015, de 10 de junio del Currículo de Bachillerato del Principado de Asturias.

Las actividades serán consecuencia de estrategias contextualizadoras que harán referencia continua al entorno social, laboral y cultural del alumno o alumna. El motor principal del proceso de enseñanza-aprendizaje lo constituye la simulación de casos reales y la proximidad de ejemplos cercanos relacionados con el contexto económico y cultural de referencia. Se trabajarán temas transversales como la educación medioambiental, educación para la paz para el consumo responsable y para la no discriminación. Las actividades tendrán como telón de fondo "la empresa" y se insistirá en temas de contaminación y sus costes, escasez de recursos naturales, deterioro de la calidad de vida, igualdad de oportunidades para ambos sexos, importancia en el cumplimiento de la normativa mercantil y laboral, entre otros.

Las actividades recogidas en esta propuesta de innovación son las siguientes:

\section{Isla Desierta:}

La base de la Economía consiste en la asignación eficiente de los recursos (que son escasos) para satisfacer las necesidades de 


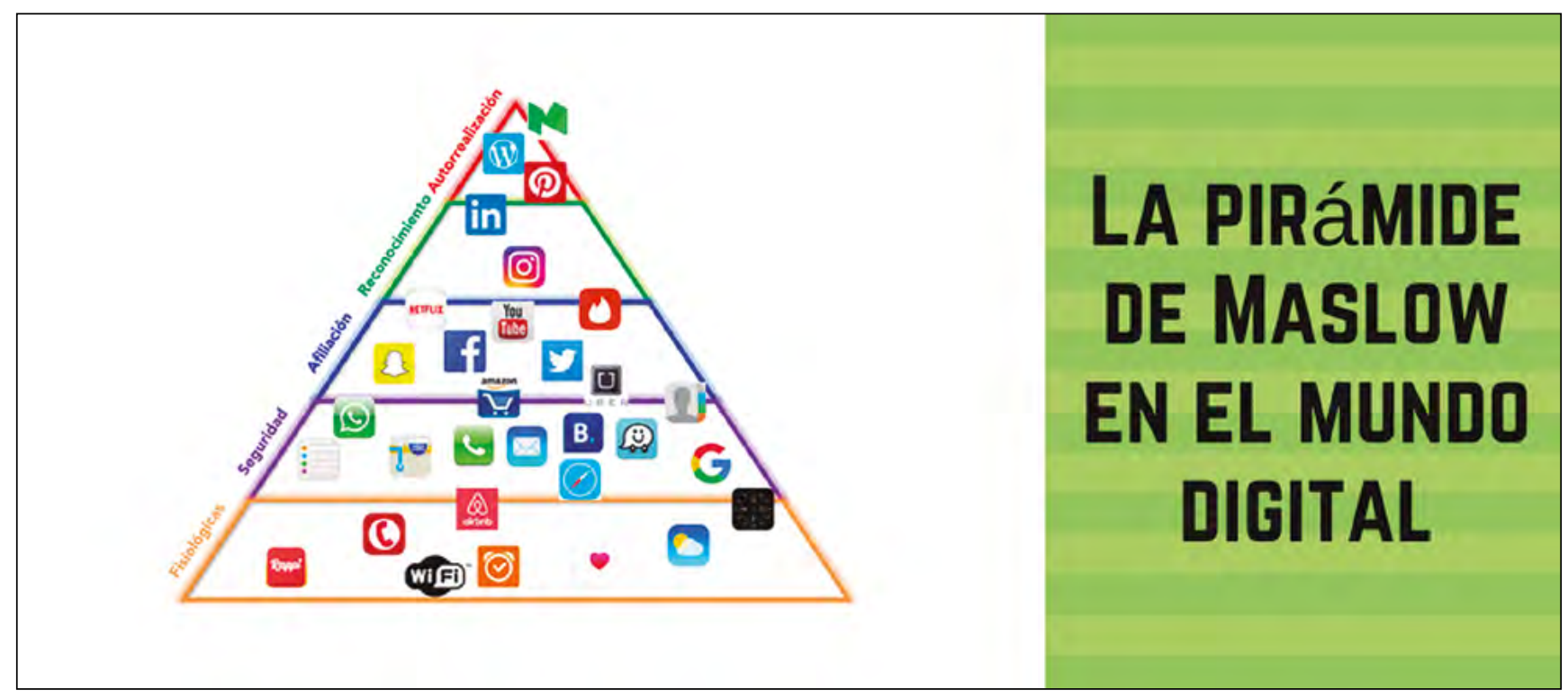

Figura 1. Pirámide de Maslow en el mundo digital (Vélez, 2017)

los individuos (que son ilimitadas). Maslow clasifica las necesidades humanas en una pirámide y las ordena jerárquicamente en cinco niveles (Quintero, 2007). El objetivo de esta actividad es demostrar al alumnado la importancia de la economía; cómo esta materia está presente en sus vidas sin darse cuenta y como todas sus decisiones tienen relación con la asignatura "Economía", en concreto, con la escasez de recursos y las necesidades ilimitadas de los individuos.

Para explicar estos contenidos, puede comenzarse realizando un experimento de aula que permita relacionar las decisiones que tomen, cada uno de los equipos, con conceptos económicos y clasificación de necesidades. Para ello, se desarrollan en el aula un juego por equipos denominado "Isla Desierta".

El desarrollo de dicho juego es tal como sigue: "Imaginad que viajáis en un avión, que sufre un accidente y todos los supervivientes del vuelo os veis atrapados en una isla desierta, de la que no os rescatarán a corto ni a medio plazo. Tras este suceso, os encontráis en una situación como la que protagonizó Robinson Crusoe en la novela Aventuras de Robinson Crusoe escrita por Daniel Defoe (1863). ¿Qué haríais?"

Los alumnos y alumnas deberán tomar decisiones de grupo. Lo lógico será que las decisiones más inmediatas estén relacionadas con cubrir las necesidades básicas o primarias y, a medida que éstas se vayan satisfaciendo, pasarán al siguiente nivel de la pirámide de Maslow. Para captar su atención, además, se trabajará con una pirámide versionada según era digital (ver Figura 1).

Con esta actividad, se pretende captar la atención del alumnado, ya que les resultará "distinta", sirviendo como primera toma de contacto a los equipos de trabajo que a lo largo de todo el curso escolar tendrán que ir colaborando.

\section{Divide y Vencerás:}

El objetivo de esta actividad es mostrar al alumnado la importancia del trabajo en equipo. Para ello, se propondrá la realización de un juego en el aula que, además de potenciar las competencias para trabajar en equipo, permita a los y las estudiantes relacionar conceptos de proceso productivo, especialización y división del trabajo. De ahí, que el nombre de la actividad sea "Divide y vencerás".

Para ello, los alumnos y alumnas deben ser divididos en grupos de 3 personas. Cada grupo representará a una empresa y todos ellos se dedicarán a la misma actividad. Por tanto, son competidores directos en el mercado de trabajo. A su vez, se debe separar a todos los grupos de estudiantes en dos bandos: uno de ellos está formado por empresas que aplican la especialización y división del trabajo en su proceso productivo; y el otro grupo estará formado por empresas en las que no hay división del trabajo: cada operario trabaja sólo y realiza todas las partes del proceso productivo de forma completa e individual.

La tarea que deben de realizar es la misma en cada empresa (para que los resultados sean comparables) y consiste en producir unos folletos, a modo de tríptico, de las empresas. Se pueden utilizar materiales trabajados en actividades previas, si los hay. En todo caso, el contenido del folleto debe incluir un título y un slogan (que serán muy similares para todas las empresas) y una imagen cortada y pegada. La idea es analizar cuántos folletos puede producir cada empresa en un tiempo determinado, por ejemplo, de 15 minutos.

A continuación, los alumnos y alumnas deben realizar una segunda ronda de producción de folletos. Y una vez que termine, se vuelven a contar el número de unidades producidas de cada grupo.

Esta actividad trata de mostrarles a los alumnos y alumnas, de manera práctica, la base teórica sobre la que se trabaja, para mostrarles la eficacia del trabajo en equipo en determinadas circunstancias. Se pretende que el alumnado entiendan la importancia de la división del trabajo (los grupos del bando 1 deberían de producir más) y la importancia de la especialización del trabajo (es previsible que en la segunda ronda se produzcan más unidades, ya que están más especializados que la primera vez).

\section{Creando empresas:}

Para hacer más atractivos los contenidos relacionados con las clasificaciones de empresas y tipos de mercados, puede ponerse en práctica una actividad basada en la simulación empresarial.

El alumnado debe formar grupos de trabajo. Cada equipo debe constituir una empresa y darle forma. La Economía centra su eje principal de actuación en la toma de decisiones, así que estos grupos de trabajo tendrán que ponerse de acuerdo, debatir sobre las ventajas e inconvenientes de cada una de las formas jurídicas de las empresas y constituir su propio negocio.

Para desarrollar esta tarea, se les debe facilitar un guion de trabajo que puede servirles de orientación en la toma de decisiones. Tras las experiencias anteriores de Isla Desierta y Divide y Vencerás, los alumnos y alumnas ya conocen el significado de trabajo en equipo, reparto de tareas y consenso en la toma de decisiones, por 
lo que debería de notarse ese grado de madurez durante el desarrollo de la actividad. Se espera que los integrantes ya trabajen más en equipo, que no en grupo, que en las actividades previas.

\section{Debates en el aula:}

Otros de los temas a trabajar en el aula podrían relacionarse con el proceso de creación y valor del dinero, e indicadores de variación. Contenidos muy actuales y presentes en las vidas de toda la ciudadanía. Es ideal para resaltar el origen del dinero como herramienta para el intercambio de bienes, su valor simbólico, su utilización como referente y la importancia de su buen uso.

Para despertar el espíritu crítico y hacer que los alumnos y alumnas profundicen en el proceso, podrían generarse debates en el aula, lo cual propiciaría la discusión de grupo y la defensa de distintos puntos de vista.

Además de generar debates en el aula, se podría realizar una aproximación a situaciones cotidianas y relevantes de la vida de los jóvenes, basándose en su entorno vital e intereses. La idea principal es que sean capaces de formar una opinión propia sobre el dinero.

Asimismo, se pueden aprovechar estas sesiones para vincular el concepto de dinero con la idea de consumo orientado hacia la sostenibilidad del planeta o también para hablar abiertamente de las, hasta hace poco, desconocidas criptomonedas, su imparable irrupción en los mercados y los riesgos aparejados a ellas.

\section{Jornadas Medioambientales:}

Con una actividad como la realización de unas Jornadas medioambientales se puede trabajar por la sostenibilidad del pla- neta y por el fomento de un consumo responsable, desde la perspectiva de la conciencia medioambiental y social, abordando una gran variedad de temas relacionados con el sector público, la regulación de la economía, fallos de mercado y, también, con economía sostenible y medio ambiente. Así mismo, pueden abordarse temas tan importantes como las desigualdades de riqueza entre países, las razones para la deslocalización de las empresas y la explotación de recursos, dando visibilidad a los países en vías de desarrollo y a los intereses empresariales y económicos ocultos.

El medio ambiente es un recurso sostenible y escaso, que se debe cuidar y trabajar en todas las etapas del sistema educativo, para generar conciencia entre las personas que cambiarán el mundo.

\section{Propuesta de evaluación}

En la mayoría de los grupos de trabajo es habitual que haya diferencias y diversidad de aportaciones al grupo (free riders), por tanto, todo grupo de trabajo debe de evaluarse de forma conjunta, pero también individualmente, ya que no todos los miembros aportan la misma cantidad y/o calidad de trabajo al grupo.

Para llevar un registro diario de cada sesión de trabajo se puede utilizar una ficha de estudiante, en la que se pueden ir reflejando las impresiones o las anotaciones que se estimen oportunas de cada uno de los miembros.

Un ejemplo de rúbrica para la evaluación del trabajo individual dentro de un equipo puede verse en la Tabla 1.

Se propone que la calificación de estas actividades de trabajo en equipo podría suponer un $20 \%$ de la nota final de la asigna-

Tabla 1. Ejemplo de rúbrica para la evaluación del trabajo individual de cada miembro de un equipo de trabajo

\begin{tabular}{|c|c|c|c|c|c|}
\hline & $\begin{array}{l}\text { Bajo aprendizaje } \\
\text { (1 punto) }\end{array}$ & $\begin{array}{l}\text { Aprendizaje medio } \\
\quad(2 \text { puntos })\end{array}$ & $\begin{array}{l}\text { Buen aprendizaje } \\
\text { (3 puntos) }\end{array}$ & $\begin{array}{l}\text { Excelente aprendizaje } \\
\text { (4 puntos) }\end{array}$ & Calificación \\
\hline$\frac{y}{5}$ & $\begin{array}{l}\text { Rara vez escucha, com- } \\
\text { parte o apoya el esfuer- } \\
\text { zo de los demás. Fre- } \\
\text { cuentemente no es buen } \\
\text { miembro del grupo. }\end{array}$ & $\begin{array}{l}\text { A veces escucha, comparte } \\
\text { o apoya el esfuerzo; pero al- } \\
\text { gunas veces no es un buen } \\
\text { miembro del grupo. }\end{array}$ & $\begin{array}{l}\text { Usualmente escucha, com- } \\
\text { parte y apoya el esfuerzo } \\
\text { de otros. No causa "pro- } \\
\text { blemas" en el grupo. }\end{array}$ & $\begin{array}{l}\text { Casi siempre escucha, apo- } \\
\text { ya y comparte el esfuerzo } \\
\text { de otros. Trata de mante- } \\
\text { ner la unión de los miem- } \\
\text { bros. }\end{array}$ & $30 \%$ \\
\hline $\mathbb{Z}$ & $\begin{array}{l}\text { Tiene una actitud nega- } \\
\text { tiva hacia el trabajo. }\end{array}$ & $\begin{array}{l}\text { Algunas veces tiene una ac- } \\
\text { titud positiva hacia el traba- } \\
\text { jo. }\end{array}$ & $\begin{array}{l}\text { Casi siempre tiene una acti- } \\
\text { tud positiva hacia } \\
\text { el trabajo. }\end{array}$ & $\begin{array}{l}\text { Siempre tiene una actitud } \\
\text { positiva hacia el trabajo. }\end{array}$ & $30 \%$ \\
\hline$\frac{\sqrt{2}}{\frac{1}{2}}$ & $\begin{array}{l}\text { No trata de resolver pro- } \\
\text { blemas o ayudar a otros } \\
\text { a resolverlos. Deja que } \\
\text { otros hagan el trabajo. }\end{array}$ & $\begin{array}{l}\text { No sugiere soluciones, pero } \\
\text { está dispuesto a tratar solu- } \\
\text { ciones propuestas por otros. }\end{array}$ & $\begin{array}{l}\text { Refina soluciones sugeridas } \\
\text { por otros compañeros/as. }\end{array}$ & $\begin{array}{l}\text { Busca y sugiere soluciones } \\
\text { a los problemas. }\end{array}$ & $20 \%$ \\
\hline 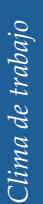 & $\begin{array}{l}\text { No ha habido intercam- } \\
\text { bio de información y } \\
\text { puesta en común en el } \\
\text { grupo. }\end{array}$ & $\begin{array}{l}\text { La puesta en común ha re- } \\
\text { sultado significativamente } \\
\text { insuficiente. }\end{array}$ & $\begin{array}{l}\text { La puesta en común ha } \\
\text { sido insuficiente. El clima } \\
\text { de trabajo en el grupo ha } \\
\text { sido adecuado. }\end{array}$ & $\begin{array}{l}\text { El clima de trabajo y las } \\
\text { actividades han sido ade- } \\
\text { cuadas. Hubo bastante in- } \\
\text { tercambio de información } \\
\text { entre compañeros. }\end{array}$ & $20 \%$ \\
\hline
\end{tabular}


1. Dar a cada componente del grupo la nota media de las puntuaciones individuales. Se evidencia así qué personas del grupo aportaron más y están por encima de la media y qué

personas restaron nota al estar por debajo de la media.
1. Dar a cada estudiante su puntuación individual más la media de su grupo. De esta manera, el influjo del trabajo de cada persona en el grupo sigue estando presente, pero en menor medida.
2. Puede optarse por dar a los miembros del grupo la calificación media obtenida de entre las puntuaciones individuales, de modo que cada estudiante vea que está aportando algo a la nota del grupo.

3. También se puede puntuar a todo el grupo con la nota del integrante que haya obtenido la calificación más baja. Así, se intenta que todos los miembros se involucren y ayuden a los compañeros/as menos aventajados.
2. Dar a cada estudiante su puntuación individual más otros puntos extra en caso de que todos los miembros hayan alcanzado los mínimos previstos. tura; ya que esta propuesta de actividades es complementaria a otros procesos de enseñanza-aprendizaje que puedan llevarse a cabo en las aulas, incluyendo sistemas tradicionales de enseñanza y sistemas clásicos de evaluación.

En cuanto a las calificaciones, existen dos formas de aplicar las mismas. Una de ellas es poniendo la misma nota a todos los miembros del equipo. Otra, poniendo distinta nota a cada miembro (Ver Tabla 2).

Cualquiera de estas posibilidades provoca que haya un esfuerzo y un interés especial en trabajar y en que los resultados sean los mejores posibles, ya que la nota de todos los miembros dependerá de ello.

Además, se adquiere una doble responsabilidad: una responsabilidad individual y una responsabilidad de cara al equipo de trabajo al que se pertenece.

Por último, en todo grupo de trabajo el desarrollo de las actividades puede descomponerse en tareas más sencillas y concretas, donde todos los miembros tienen posibilidades de colaborar en función de sus habilidades y capacidades para aportar buena nota al grupo. Ello conlleva una mejora en su autoestima y en la consideración que de ellos se tiene en general (Gavilán, 2019).

\section{Conclusiones}

Los y las adolescentes necesitan fuentes de motivación que les estimulen en su día a día, que les impulsen a explotar las oportunidades que les brindan los centros educativos, para exprimir al máximo todas sus habilidades y capacidades.

Numerosos estudios con base científica respaldan las ventajas del trabajo en equipo, incidiendo de forma directa en los resultados y observando una mejora del rendimiento del grupo, la motivación en términos individuales, $\mathrm{y}$, en consecuencia, la productividad de todo el conjunto de la clase.

Además, el trabajo en equipo puede ser un buen aliciente para estimular las necesidades de superación de los alumnos y alumnas, su autoestima, su sentido de realización y de pertenencia al grupo y, puesto que somos seres sociales, a mejorar bienestar personal de los individuos.

Por lo tanto, trabajar en equipo puede motivar enormemente al alumnado, a la par que le puede ayudar a desarrollar una competencia clave: "aprender a aprender", encaminada a mejorar la habilidades sociales y cívicas y al desarrollo del pensamiento personal y del espíritu crítico.

\section{Agradecimientos}

Este trabajo ha sido desarrollado sin recibir financiación de ninguna entidad, agencia $\mathrm{u}$ organismo oficial.

\section{Referencias}

Arís, N. (2009). El síndrome de Burnout en los docentes. Electronic Journal of Research in Educational Psychology, 7(2), 829-848.

Barraycoa, J. y Lasaga, O. (2010). La competencia de trabajo en equipo: más allá del corta y pega. Universidad de La Rioja. Vivat Academia, 111, 65-69.

Cardona, P. y Wilkinson, H. (2006). Trabajo en Equipo. Recuperado de: https://media.iese.edu/research/pdfs/OP-07-10-E.pdf

Chicharro, J.M., Jerez, O. y López, M. (2015). Experiencias de innovación docente en enseñanza superior de Castilla y La Mancha. Recuperado de: HYPERLINK “https://ruidera.uclm.es/ xmlui/bitstream/handle/10578/7352/Experiencias_de_innovaci\%C3\%B3n_2015.pdf?sequence $=1 \&$ is Allowed $=y$ Ciudad\%20Real." https://ruidera.uclm.es/xmlui/bitstream/handle/10578/7352/Experiencias_de_innovaci\%C3\%B3n_2015. pdf?sequence $=1 \&$ isAllowed=yCiudad Real.

Defoe, D. (1863). Aventuras de Robinson Crusoe. Murcia y Marti Editores.

Ferrán, P., González, M., García, Y., Malo, S., y Casas, F. (2015). Los estilos y estrategias de afrontamiento y su relación con el bienestar personal en una muestra de adolescentes. Anales de la Psicología, 31(1), 226-233

Gavilán, P. (s.f.). El trabajo cooperativo: una alternativa eficaz para atender a la diversidad. Recuperado de: http://conexiones.dgire.unam.mx/wp-content/uploads/2017/09/El-trabajo-cooperativo-una-alternativa-eficaz-para-entender-la-diversidad.pdf

Gil, P.R. (2005). El sindrome de quemarse por el trabajo (burnout). Una enfermedad laboral en la sociedad del bienestar. Ediciones Pirámide.

Guitert, M., Romeu, T. y Pérez-Mateo, M. (2007). Competencias TIC y trabajo en equipo en entornos virtuales. Revista de Universidad y Sociedad del Conocimiento, 4(1), 1-12. http://dx.doi. org/10.7238/rusc.v4i1.289

Gutiérrez, D. (2009). El síndrome de Burnout en alumnos de educación secundaria. Investigación Educativa Duranguense, 10, 1-10. Recuperado de: https://dialnet.unirioja.es/servlet/articulo? codigo $=2941614$ 
Hernández, L.C. y Hernández, E.H. (2015). La motivación y el trabajo en equipo. República de Cuba. Recuperado de: https://www. academia.edu/21085889/LA_MOTIVACI\%C3\%93N_Y_EL_ TRABAJO_EN_EQUIPO

Herrazo, J. y Moriana, J.A. (2004). Estrés y Burnout en profesores. International Journal of Clinical and Health Psychology, 4(3), 597-621.

Marín, J.A. y Zarate, M.E. (2008). Propuesta de un modelo integrador entre la gestión del conocimiento y el trabajo en equipo. Intangible Capital, 4(4), 255-280.

Mejía, A., Pastrana, J.J. y Mejía, J. (2011). La autoestima, factor fundamental para el desarrollo de la autonomía personal y profesional. XII Congreso Internacional de la Teoría de la Educación CITE 2011. Recuperado de: https://www.cite2011.com/Comunicaciones/A+R/103.pdf

Palomo, R., Ruiz, J. y Sánchez, J. (2006). Las TIC como agentes de innovación educativa. Recuperado de: https://www.observatoriodelainfancia.es/oia/esp/documentos_ficha.aspx?id=1112

Pérez, G., Al Pineda, U. y Arango, M. D. (2011). La capacitación a través de algunas teorías de aprendizaje y su influencia en la gestión de la empresa. Revista Virtual Universidad Católica del Norte, 33, 1-22

Poy, R., Mendaña, C. y González, B. (2015). Diseño y evaluación de un juego serio para la formación de estudiantes universitarios en habilidades de trabajo en equipo. RISTI - Revista
Ibérica de Sistemas e Tecnologías de Informaçao, 3, 71-83. http:// 10.17013/risti.e3.71-83.

Quintero, J.R. (2007). Teoría de las Necesidades de Maslow. Recuperado de: https://paradigmaseducativosuft.blogspot.com/search?q=maslow

Ricoy, C.J. (2005). La Teoría del Crecimiento Económicode Adam Smith. Universidad de La Habana. La Habana: Economía y Desarrollo, 138(1), 11-47.

Suárez, J., Maiz, F. y Meza, M. (2010). Inteligencias Múltiples: Una innovación pedagógica para potenciar el proceso enseñanza aprendizaje. Investigación y Posgrado, 25(1), 81-94.

Torrelles, C., Coiduras, J., Isus, S., Carrera, F.X., Paris, G. y Cela, J.M (2011). Competencia de trabajo en equipo: Definición y Categorización. Revista de Currículum y Formación del Profesorado, 5(3), 330-344.

Vélez, F. (2017). La pirámide de Maslow en el mundo digital. Recuperado de: https://www.linkedin.com/pulse/la-pir\%C3\%A1mide-de-maslow-en-el-mundo-digital-felipe-v\%C3\%A9lez-gonz\%C3\%A1lez/?originalSubdomain=es

Viñas, F., González, M., García, Y., Malo, S. y Casas, F. (2014). Los estilos y estrategias de afrontamiento y su relación con el bienestar personal en una muestra de adolescentes. Anales de Psicología, 31(1), 226-233. https://doi.org/10.6018/analesps.31.1.163681 